\title{
Design of Monitoring System of High Temperature Piping System by Heat Resistant Fiber Bragg Grating
}

\author{
Yukihiro SHIMADA $^{* 1}$, Akihiko NISHIMURA ${ }^{* 1}$, Masanari YOSHIKAWA ${ }^{* 2}$ and Takao KOBAYASHI ${ }^{* 2}$ \\ ${ }^{* 1}$ Kansai Photon Science Institute, JAEA, 8-1 Umemidai, Kizugawa-shi, KYOTO, 619-0215, Japan \\ E-mail: shimada.yukihiro@jaea.go.jp \\ ${ }^{* 2}$ Graduate School of Engineering, University of Fukui, 3-9-1 Bunkyo, Fukui-shi, \\ FUKUI, 910-8507, Japan
}

\begin{abstract}
Ultrafast laser processing was applied to fabricate fiber Bragg gratings with specific wavelength characteristics. The high-temperature properties were examined. Reflection was not changed greatly when it was exposed to temperature of up to $600^{\circ} \mathrm{C}$ for 1 hour. A time-domain detector of FBG was produced for vibration measurement. FBG of three wavelength was processed in one optical fiber, and the possibility of the multipoint measurement was shown. It was proposed that the heat-resistant FBG sensors combined with wavelength tunable laser will become powerful tool in surveillance for coolant piping sys-tem in nuclear power plants.
\end{abstract}

DOI: $10.2961 / \mathrm{jlmn} .2010 .01 .0021$

Keywords: fiber Bragg grating, point-by-point method, seismic safety, nuclear power plant

\section{Introduction}

In the case that large-scale factories have complex piping systems, both aging management and seismic safety are indispensable. Upgrading state surveillance technologies are necessary to avoid serious accidents [1]. For instance, in seismic safety research, surveillance for piping system is especially important in nuclear power plants. Various assessment methods are actively discussed in the recent earthquake [2].

A fiber Bragg grating (FBG) sensor is very useful in surveillance for complex piping system [3]. In nuclear power plants, it can monitor both temperature and strain in the complex piping system with remote condition [4]. However, the FBG sensor has the fault that it is not possible to use it at the high temperature, because a usual FBG sensor is made by the UV interference exposure [5]. On the other hand, this technology was introduced from becoming of high precision work to making material such as glass and ceramics possible by the development of femtosecond pulse laser fabrication [6]. Kondo and Hirao et. al. succeeded in writing long period fiber grating by femtosecond pulse laser. The grating structure in fiber had an excellent thermal quality, and stability up to $500{ }^{\circ} \mathrm{C}$ was confirmed [7]. And the production of FBG with the point-by-point method succeeded by Martinez et. al. [8]. Moreover, the high temperature tolerance examination was done by Grobnic et.al., and stability up to $1000{ }^{\circ} \mathbf{C}$ was reported [9].

This paper presents an improved fabrication system for FBGs, and reports on the outcome of an experiment that attempts the performance gain as the strain sensor. In addition, we reports construction of a variable wavelength fiber laser based light source for vibration measurement.

\section{Experiment}

The femtosecond laser system was a Ti:sapphire CPA laser, which can generate $100 \mathrm{fs}$ pulse duration with $10 \mathrm{~mJ}$ pulse energy at $10 \mathrm{~Hz}$ reputation. The laser system has a femtosecond oscillator, a regenerative amplifier and a 4pass amplifier. The femtosecond pulse duration was monitored by a oscilloscope. The pulse energy at the regenerative amplifier was $1 \mathrm{~mJ}$ at maximum. At the input of the 4pass amplifier, an air-gap polarizer and a 1/2 lambda waveplate are put in to attenuate the pulse energy. A Faraday isolator was inserted to protect the oscillator.

Figure 1 is a photograph in around of sample in the laser processing system. Local refractive index change by ultrafast laser processing can be observed by a phase contrast microscope. A dielectric mirror in the microscope can axially combine the laser beam and the target image. A CCD camera mounted on the microscope enable to monitor local refractive index change during the processing.

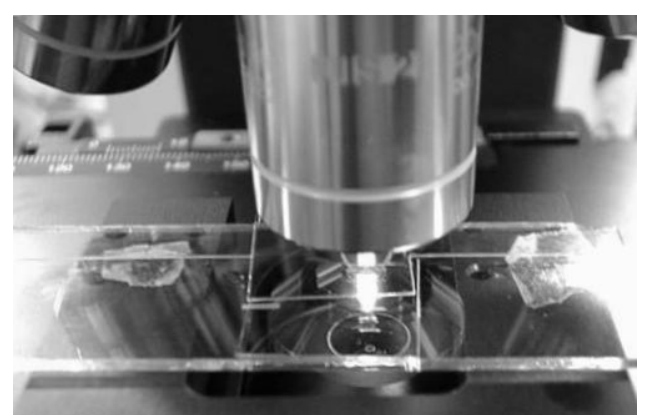

Fig.1 Experimental setup for FBG processing by CPA pulse laser. 


\subsection{High temperature tolerance examination with a quartz disk}

The periodic refractive-index change caused in a transparent medium can be evaluated because of the Raman-Nath diffraction [10]. We processed quartz disk sample as an operation check of the experiment system. A quartz disk was held at a precision stage. The quartz disk has a $12.5 \mathrm{~mm}$ diameter with $3 \mathrm{~mm}$ thickness. The precision stage can be controlled by high accuracy of $10 \mathrm{~nm}$ in order to evaluate degradation on the quartz disk under high temperature.

Figure 2(a) shows the phase contrast microscope image of the processing sample. The vertical direction in the figure is $5 \mu \mathrm{m}$, and the horizontal direction is $10 \mu \mathrm{m}$ at intervals of the processing. The processing shape of this sample is compared to the sample by converging the He-Ne laser on the vertical, and measuring the contrast of the diffraction light image that appears at the back.

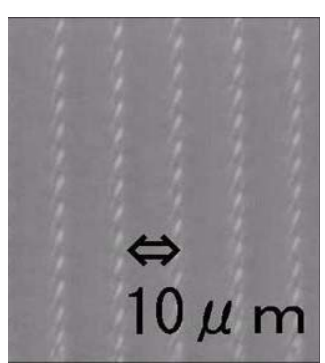

(a)

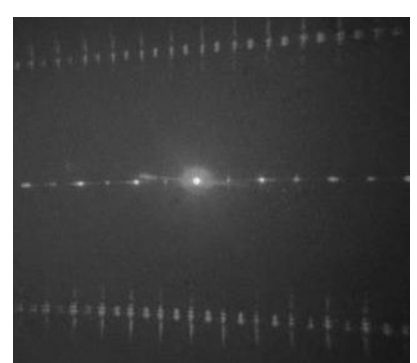

(b)
Fig. 2 Laser processing pattern and diffraction pattern

(a) Phase contrast microscope image,

(b) Temperature tolerance check by diffraction pattern

Figure 2(b) shows the diffraction light image. The hightemperature testing of the sample was heated in the vacuum bell jar, and the change was measured in the contrast of the diffraction light image before and after heating. As a result of no change in the contrast of the diffraction ray after overheating of $950^{\circ} \mathrm{C}$ and $1 \mathrm{~h}$, it was confirmed that this processing had high temperature tolerance.

\subsection{Search for the optimum conditions}

The disorder of the diffraction pattern of Fig. 2(b) originates in the laser beam machining shape. Therefore, the search for the best laser optical conditions is as follows. Figure 3 shows the change in the processing shape by the difference of the pulse energy of the laser. As for the energy of the light used for the processing from this result, it has been understood that about $1 \mu \mathrm{J}$ is best.

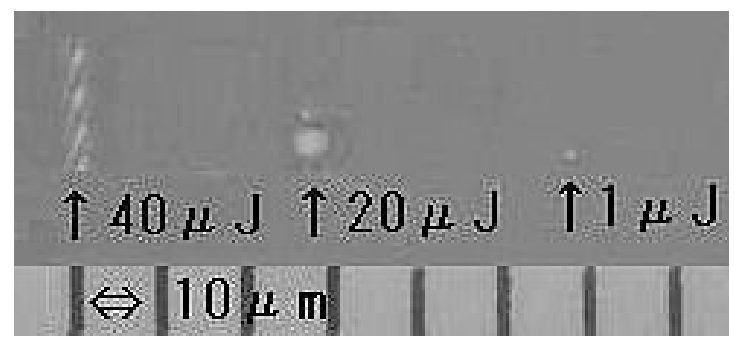

Fig. 3 The change in the processing shape by the difference of the pulse energy of the laser
The object processed in the core of a diameter of $10 \mu \mathrm{m}$ or less is indispensable to the processing of FBG by the pointby-point method. The following techniques were used. The optical fiber sample was made and the side on which the laser will be incident was made plane by filling with a refractive index adjustment liquid.

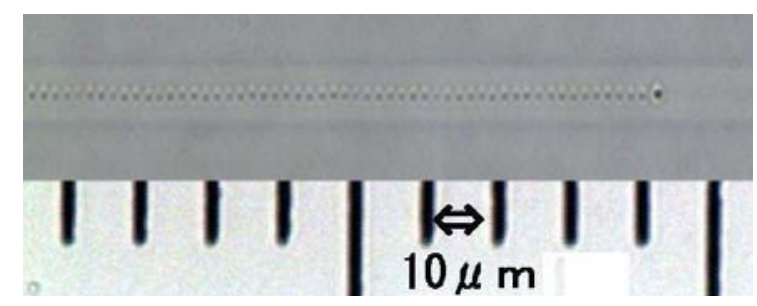

Fig. 4 Phase contrast microscope image of processed optical fiber

\subsection{Fabricating an FBG in an optical fiber}

We created an FBG structure on the side of the optical fiber using a point-by-point method. Figure 4 shows a photograph of the FBG in optical fiber. It is shown the processing part a consecutive points, and to be processed to the center of core. The core diameter of the single-mode optical fiber used was $8 \mu \mathrm{m}$, and the cladding diameter was 125 $\mu \mathrm{m}$. It is necessary to fabricate the FBG accurately in the core. Because a phase microscope can measure minute differences in refractive index, a correct processing to a core of the optical fiber and can a distinction of the part of clad, and a horizontal core becomes possible. However, focusing in the direction of the incidence of the light is not easy.

The normal angle of incidence degree changes from the difference of the refractive index when the laser light enters into the sample. For instance, the refractive indexes of the optical fiber are $n=1.45$, air is $n=1.0$. Because of the cylindrical shape of the optical fiber, the focus position in the direction of the long axis and the direction of the circumference are different. Then, the processing shape of the focus doesn't become as true globular. There are two following methods to correct this thing. One method is a thing to change the divergence of incident light by two orthogonal directions, and it is possible to achieve it by setting up a cylinder of long focus lens in front of the microscope.

However, there is a necessity for changing the divergence depending on the focal length of object lens to achieve this method. Another method is a method of filling cylinder sample surroundings with the refractive index adjustment liquid equal with the optical fiber. The refractive index of the 1-bromonaphthalene used this experiment is $\mathrm{n}=1.52$, and a value close to the optical fiber deflecting. Because the anisotropy of the refractive index of the optical fiber decreases if it does so, it only has to make the divergence of incident light an isotropic. We processed optical fiber by using a latter method. The level on the refractive index adjustment liquid in surroundings of the fiber sample is influenced easily for the flow of air and the vibration of sample table. Moreover, because the laser enters into this respect, swinging of the solution level changes the focus shape of the laser. Then, we suppressed the above issue by covering the face where the laser entered as shown in Fig.1 with the cover glass. We observed the super white light 
generated when processing optical fiber and optimized the laser light irradiation. The generated white light reaches the roller end face through the fiber if the irradiation is done in the core. White light doesn't spread if the core is removed and the irradiation is done on a clad inside. The signal strength is monitored with the oscilloscope by setting up the photo-multiplier in the roller end face of the fiber. The dichroic filter that suppresses the light of the processed wavelength is set up on the fiber edge side, and only the white light caused as a result of the processing is entered into the photo-multiplier. Whether it is processed from strength of the generated white light to the core of the fiber by these methods can be confirmed. It is possible to construct at a low price compared with the method of using the spectroscope, and it is possible to execute it even by the processing use of high repetition rate laser. The specimen support that maintains the optical fiber was moved with a stepping motor stage using a harmonic geared drive. Once the closed loop control is done, the sample stage was driven by an accuracy of $10 \mathrm{~nm}$ or less.

\subsection{Evaluation of reflection wavelength characteristic and heat tolerance}

An evaluation of characteristics of processed FBG has gone to measure the reflected spectrum. White light source was connected with FBG through the circulator, and the reflected light was measured with the spectroscope.

Figure 5 shows the result of doing the heating examination of FBG. The processing interval is $1590 \mathrm{~nm}$, and this corresponds to the third Bragg wavelength. The processing part is 50 points, and the total processing length is about $80 \mu \mathrm{m}$. The change in reflectivity was seen from $1543 \mathrm{~nm}$ on the long-wavelength side as a result of subjecting this sample to the heating examination for $600^{\circ} \mathrm{C}$ and 24 hour, and comparing the reflected spectra before and after the temperature rise. However, substantial change was not seen in the reflectivity at about $1538 \mathrm{~nm}$, which was the designed wavelength, and the FBG structure was maintained to the extent that its practical use was confirmed.

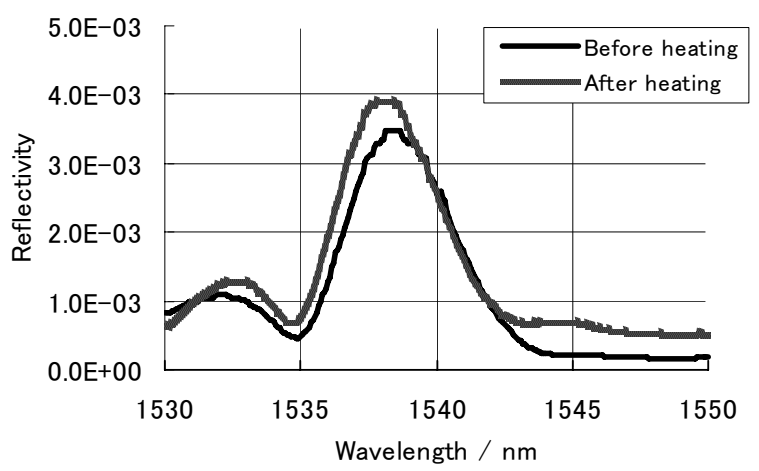

Fig. 5 Comparison of FBG spectra before and after heating

\subsection{Multiplexing of FBG processing wavelength}

A FBG sensor system has the advantage that many can measure points. It is necessary to multiplex the characteristic wave length for that. At the UV light exposure method, it is necessary the mask for exposure matched to the characteristic wave length. FBG of arbitrary characteristic wave length can be made only from changing the moving speed of the sample stage in this point by point method.

Figure 6 shows the reflected spectrum of the optical fiber in which the FBG structure of three wavelength is written. Three wavelength is $1543,1563,1583 \mathrm{~nm}$, and it processes in the place where $200 \mathrm{~mm}$ interval is different. In the processing place of each part, the length of 400 points and the total processing is about $600 \mu \mathrm{m}$. The FWMH wavelength of the FBG reflection spectrum is $2 \mathrm{~nm}$. We can measure about 40 points if it seems that the gain region of the measurement source of light is $80 \mathrm{~nm}$ [11]. The cause of broad peak is Fresnel reflection from the side of the fiber edge, and spectrum of the white light source used for the measurement is shown.

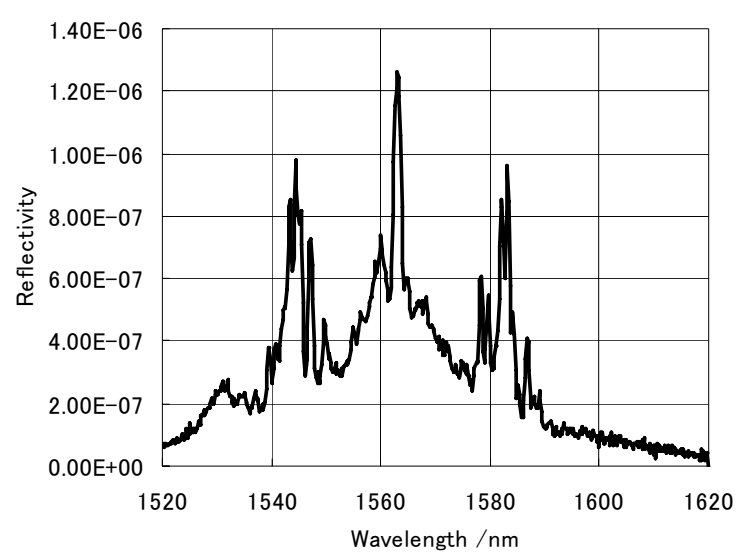

Fig. 6 Spectrum of FBG that writes three wavelength

\section{Conclusion}

Femtosecond pulse laser processing could fabricate grating structures in a quartz disc and a fiber core. In the case of quartz disc, it seemed that the grating structure was free form degradation at $950{ }^{\circ} \mathrm{C}$ by the diffraction pattern check. In addition of index matching liquid for a optical fiber, a FBG was successfully fabricated. Although the FBG was confirmed to be heatproof up to $600^{\circ} \mathrm{C}$ by hightemperature testing, the peak shift on reflection spectra of the FBG was slightly observed. Multiple FBG with $20 \mathrm{~nm}$ wavelength separation was demonstrated. The multiple FBG fabricated by ultrafast laser processing can work as heat-resistant strain sensors. Combining them with a tunable fiber laser, we are going to demonstrate the statesurveillance or structural health-monitoring for seismic safety on nuclear power plants.

\section{Acknowledgement}

The authors wish to thank Mr. Motoo Doi for his continuous effort in research planning and Mr. Takashi Masuzumi for his technical assistants in heating test on the fabricated FBG. And the authors also thank Dr. Koichi Saruta and Dr. Kazuyuki Tsukimori for valuable discussions in seismic safety and advanced maintenance about Fast Breeder Reactors. 


\section{References}

[1] H. Suzuki: J. J. Soc. Non-destructive Inspection, 58, (2009) 175. (in Japanese)

[2] H. Nariai: Nuclear Safety Research Forum, (NSC, MEXT and NISA, Tokyo, 2008) pI-62.

[3] A. Othonos: Rev. Sci. Instrum., 68, (1997) 4309.

[4] K. Matsuba, C. Ito. H. KAWAHARA and T. Aoyama: J. Power and Energy Systems, 2, (2008) 545.

[5] K. O. Hill, Y. Fujii, D. C. Johnson, and B. S. Kawasaki: Appl. Phys. Lett., 32, (1978) 647.

[6] K. M. Davis, K. Miura, N. Sugimoto, and K. Hirao: Opt. Lett., 21, (1996) 1729.
[7] Y. Kondo, K. Nouchi, T. Mitsuyu, M. Watanabe, P. G. Kazansky, K. Hirao: Opt. Lett., 24, (1999) 646.

[8] A. Martinez, M. Dubov, I. Khrushchev, I. Bennion: Electron. Lett., 40, (2004) 1170.

[9] D. Grobnic, C. W. Smelser, S.J.Mihailov and R.B.Walker: Meas. Sci. Technol., 17, (2006) 1009.

[10] T. Tamaki, W. Watanabe, K. Itoh: JLMN, 2, (2007) 26

[11] T.Kobayashi, Y. Enami, H. Ishikawa: 18th Int. Optical Fiber Sensors Conf., (OSA, Mexico, 2006), TuE12.

(Received: October 13, 2009, Accepted: February 1, 2010) 